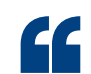

Jabba acts

as a histonedocking protein, recruiting

histones to lipid droplets

by direct physical interactions

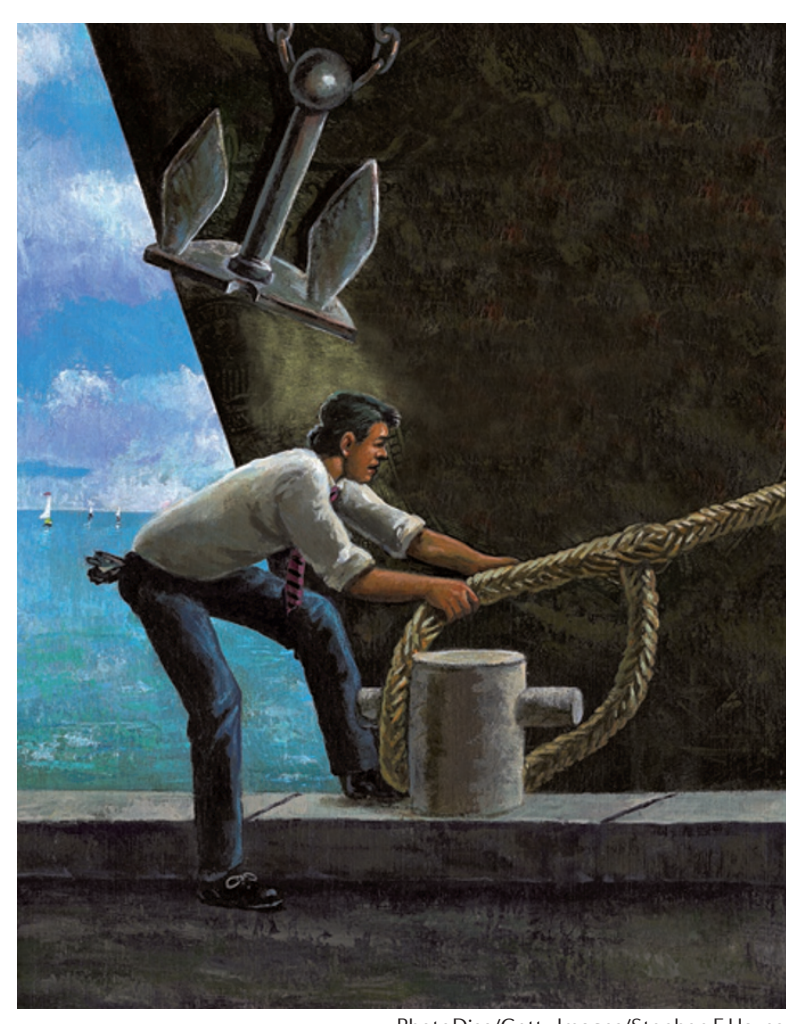

PhotoDisc/Getty Images/Stephen F Hayes

Significant levels of extranuclear histones are known to accumulate on lipid droplets, and this is thought to prevent excess histones from affecting cellular processes. However, the mechanisms that eliminate excess histones in this manner had been unclear. Here Li et al. investigate histone binding to lipid droplets in Drosophila melanogaster embryos.

The authors had previously shown that histones $\mathrm{H} 2 \mathrm{~A}$ and $\mathrm{H} 2 \mathrm{~B}$ are present in high levels on lipid droplets of early embryos. This raises the possibility that histones are bound to the droplet surface via specific protein anchors. If true, then

\title{
A histone anchor on lipid droplets
}

with H2A.v-GFP. On the basis of this, the authors propose that Jabba acts as a histone-docking protein, recruiting histones to lipid droplets by direct physical interactions.

Finally, the authors observed that the extra-nuclear pool of histones that is normally present in D. melanogaster embryos was missing in Jabba mutants, which suggests that lipid droplets are necessary to protect these histones from degradation. By manipulating the expression of histones, the authors were also able to show that sufficient histone biosynthesis is necessary for Jabba-deleted embryos to survive, supporting the biological significance of histones on lipid droplets.

The authors speculate that lipid droplets may provide a storage facility for histones and other proteins destined for other cellular compartments. Indeed, the fact that diverse proteins, including heat shock protein 70 (HSP70) and inosine monophosphate dehydrogenase, have also been reported to accumulate in lipid droplets under certain conditions suggests that lipid droplets have a general role in sequestering and inactivating proteins, facilitate protein complex assembly, promote protein delivery or aid protein degradation.

Bryony Jones reduced levels of these histones on lipid droplets. Furthermore, purification of lipid droplets from embryos expressing GFP-tagged H2A.v revealed that Jabba co-precipitates
ORIGINAL RESEARCH PAPER Li, Z. et al. Lipid droplets control the maternal histone supply of Drosophila embryos. Curr. Biol. 17 Oct 2012 (doi:10.1016/j.cub.2012.09.018) 